\title{
Use of the Response Assessment in Neuro-Oncology (RANO) criteria in clinical trials and clinical practice
}

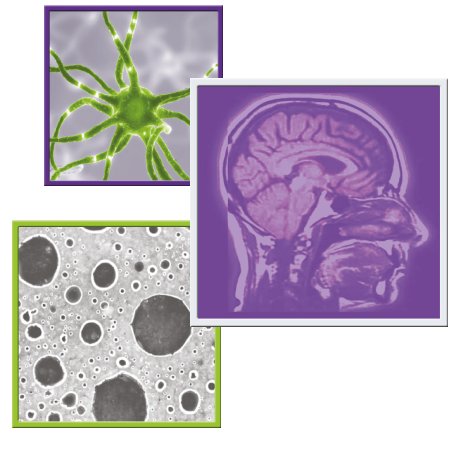

\author{
Ugonma N Chukwueke ${ }^{1,2}$ \& Patrick Y Wen*,1,2 \\ ${ }^{1}$ Department of Medical Oncology, Center for Neuro-Oncology, Dana-Farber Cancer Institute, Boston, MA, 02215, USA \\ ${ }^{2}$ Department of Neurology, Harvard Medical School, 450 Brookline Avenue, Boston, MA, 02215, USA \\ *Author for correspondence: Tel.: +1 617632 6177; Fax: +1 617632 4883; Patrick_Wen@dfci.harvard.edu
}

First draft submitted: 30 April 2018; Accepted for publication: 15 August 2018; Published online: 26 February 2019

Keywords: bevacizumab $\bullet$ CNS tumors $\bullet$ clinical trials $\bullet$ immune checkpoint inhibitors $\bullet$ pediatric brain tumor imaging • pseudoprogresion $\bullet$ pseudoresponse $\bullet$ RANO • response criteria

Between 2010 and 2014, the incidence rate of primary brain tumors in persons (aged $>20$ years) was 29.2 per 100,000 ; in children (aged <20 years), the rate was 5.81 per 100,000 [1]. Metastatic CNS tumors are known to be the most commonly occurring malignancy of the brain, although reporting for this disease is limited. Likely owing to multiple factors including improving survival from systemic malignancies, better tolerability of treatments, as well as timely and effective integration of supportive care, the incidence of CNS metastatic disease is expected to continue to increase [2]. Newer therapies and emphasis on clinical trial enrollment has made the need for effective approaches to assessing disease response even more critical.

The Response Assessment in Neuro-Oncology (RANO) working group was established to improve the assessment of tumor response and selection of end points, specifically in the context of clinical trial [3]. There has been an evolution in determining which endpoints and criteria are most important in determining therapeutic response, specifically with advances in imaging modalities. In the era of computed tomography (CT), Levin et al. conducted a retrospective analysis of 100 brain tumor patients, in which they reviewed the predictive value of specific factors and its impact upon response to treatment. In this study, the combination of radionuclide and CT scans, as well as diligent monitoring of changes in dexamethasone dose were thought to be predictive of clinical deterioration and response to chemotherapy [4]. In the following decades, the field of neuro-oncology relied upon methods derived from the extracranial solid tumor oncology, notably the MacDonald criteria and the Response Evaluation Criteria in Solid Tumors (RECIST), both methods presenting shortcoming and challenges to effective response assessment in CNS tumors. In 1990, the MacDonald criteria were proposed as the standard for assessment of response and progression, specifically in patients with high-grade glioma. These criteria used the product of the maximal perpendicular diameters but also incorporated changes in corticosteroid doses as well as neurologic function [5]. In this scheme, adopting standards from medical oncology, four categories were recommended: complete response, in which there is disappearance of all enhancing disease concomitant with neurological improvement or stability AND absence of steroids, partial response or $\geq 50 \%$ reduction in enhancing disease as well as stable neurologic status and steroid use; progressive disease (PD) or $\geq 25 \%$ increase in enhancing disease or worsening neurologic status in the setting of stable or increasing steroid use and last, stable disease (SD) defined as all other scenarios [5]. RECIST was used occasionally for evaluation of treatment response in primary and metastatic brain tumors but most brain tumor trials used the MacDonald criteria preferentially, since it was felt that use of two orthogonal diameters (2D) may have advantages over measurement of a single, longest diameter (1D) for irregularly shaped brain tumors [5].

Among the challenges to use of earlier response criteria include lack of guidance on pseudoprogression, pseudoresponse and nonenhancing tumor progression. Historical challenges in the field have also been concerned around appropriate surrogates of response and endpoints [6]. RANO working groups was established to address some of these issues and provide guidance on assessment of response and endpoints in neuro-oncology clinical trials. Although the work of RANO initially focused on gliomas, its work has extended to many other areas of neuro-

Future Medicine 
Table 1. Summary of current response criteria.

\begin{tabular}{|c|c|c|c|}
\hline Criterion & RECIST & MacDonald & RANO \\
\hline Measurement & 1D contrast enhancement & 2D contrast enhancement & $2 \mathrm{D}$ contrast enhancement $+\mathrm{T} 2 /$ FLAIR \\
\hline Progression & $\geq 20 \%$ increase in sum of lesions & $\begin{array}{l}\geq 25 \% \text { increase in product of } \\
\text { perpendicular diameter }\end{array}$ & $\begin{array}{l}\geq 25 \% \text { increase in product of } \\
\text { perpendicular diameter }\end{array}$ \\
\hline Durability of response & Optional & Yes (at least 4 week) & Yes (at least 4 week) \\
\hline Definition of measurability & Yes & No & Yes \\
\hline T2/FLAIR & Not evaluated & Not evaluated & Evaluated \\
\hline Corticosteroids considered & No & Yes & Yes \\
\hline Clinical status considered & No & Yes & Yes \\
\hline Pseudo-progression considered & No & No & Yes \\
\hline
\end{tabular}

oncology including brain metastases (BM), meningioma, pediatric tumors, spinal metastases and leptomeningeal disease.

This review will discuss the recommendations of the various RANO working groups. Although the primary focus of the RANO effort is to improve the conduct of clinical trials, some of the recommendations may be useful in the routine care of brain tumor patients.

\section{RANO-high grade glioma}

Identification of durable therapies for high-grade glioma has remained elusive to date. Despite best efforts, the standard of care for newly-diagnosed glioblastoma is unchanged, incorporating temozolomide and radiation for 6 weeks, followed by a minimum of 6 months of single-agent temozolomide [7], and possibly the addition of tumor treating fields [8]. Despite improvements in supportive care over the past decade, survival outcomes remain dismal, with 1-year survival of 39.7\% and 5-year survival of 5.5\% [1]. Endeavors to identify more effective and durable agents are ongoing in clinical trials. Challenges to drug development for high-grade glioma are multiple, including but not limited to few molecular targets, lack of reproducible preclinical models, tumor heterogeneity, poor access of agents across the blood-brain barrier and a relatively small patient population [9-11]. Further hindering development, historically, has been the inadequacy of clear and widely-accepted endpoints in the design of neurooncology clinical trials, leading to the inception of the earliest of the RANO working groups, RANO-high grade glioma (RANO-HGG).

Although the RECIST criteria is widely used for assessing response to therapy for systemic cancers [6], its use in neuro-oncology has been limited because concerns that 1D measurements may not accurately measure the irregular or asymmetric margins characteristic of HGG.

The MacDonald criteria for response assessment in high-grade glioma were first published in 1990. In comparison to RECIST, MacDonald use 2D assessments of tumor size, obtained on either contrast-enhanced CT or MRI and based upon the WHO oncology response criteria. Clinical status and use of corticosteroids was also incorporated into these criteria [5]. Representing an advance in the field, MacDonald criteria were used in early clinical trials, allowing for comparison of response rates (Table 1) [10].

With development and growing use of anti-angiogenic agents that affect vascular permeability and contrast enhancement, the limitations and shortcomings of response criteria, such as RECIST and MacDonald, has become more apparent and necessitated changes to these criteria [12]. Similar to RECIST, difficulty in measurement of irregular borders is not overcome by MacDonald. Agents such as bevacizumab and other anti-angiogenic drugs in its class are known to reduce contrast enhancement, contributing to high response rates and prolonged progressionfree survival; however, without impact in extending overall survival [11]. This is now known as 'pseudoresponse,' where the decrease in contrast enhancement is secondary to changes to vascular permeability, rather than a direct reduction in tumor size [13]. To address this issue, the RANO-HGG criteria requires that responses should be relatively durable and confirmed by a repeat MRI at least 4 weeks later [3].

The use of agents such as bevacizumab that reduce vascular permeability also introduced the problem of nonenhancing tumor progression that confounds the interpretation of PD. In up to $40 \%$ of patients treated 
Table 2. Criteria for response assessment incorporating MRI and clinical factors.

\begin{tabular}{|c|c|c|c|c|}
\hline Criterion & CR & PR & SD & PD \\
\hline $\mathrm{T} 1-\mathrm{Gd}+$ & None & $\geq 50 \% \downarrow$ & $<50 \% \downarrow$ to $<25 \% \uparrow$ & $\geq 25 \% \uparrow^{\dagger}$ \\
\hline T2/FLAIR & Stable or $\downarrow$ & Stable or $\downarrow$ & Stable or $\downarrow$ & $\uparrow^{\dagger}$ \\
\hline New lesion & None & None & None & Present $^{\dagger}$ \\
\hline Corticosteroids & None & Stable or $\downarrow$ & Stable or $\downarrow$ & $N A^{\ddagger}$ \\
\hline Clinical status & Stable or $\uparrow$ & Stable or $\uparrow$ & Stable or $\uparrow$ & $\downarrow^{\dagger}$ \\
\hline Requirement for response & All & All & All & Any $\ddagger$ \\
\hline $\begin{array}{l}\text { Summary of HGG response } \\
\text { criteria }\end{array}$ & $\begin{array}{l}\text { Requires all of the following: } \\
\text { complete disappearance of all } \\
\text { enhancing measurable and } \\
\text { nonmeasurable disease } \\
\text { sustained for at least } 4 \text { weeks; } \\
\text { no new lesions; stable or } \\
\text { improved nonenhancing } \\
\text { (T2/FLAIR) lesions; patients } \\
\text { must be off corticosteroids (or } \\
\text { on physiologic replacement } \\
\text { doses only); and stable or } \\
\text { improved clinically. Note: } \\
\text { Patients with nonmeasurable } \\
\text { disease only cannot have } \\
\text { achieved CR; the best response } \\
\text { possible is SD }\end{array}$ & $\begin{array}{l}\text { Requires all of the following: } \\
\geq 50 \% \text { decrease compared with } \\
\text { baseline in the sum of products } \\
\text { of perpendicular diameters of } \\
\text { all measurable enhancing } \\
\text { lesions sustained for at least } \\
4 \text { weeks; no progression of } \\
\text { nonmeasurable disease; no } \\
\text { new lesions; stable or improved } \\
\text { nonenhancing (T2/FLAIR) } \\
\text { lesions on same or lower dose } \\
\text { of corticosteroids compared } \\
\text { with baseline scan; the } \\
\text { corticosteroid dose at the time } \\
\text { of scan evaluation should be no } \\
\text { greater than the dose at time } \\
\text { of baseline scan; and stable or } \\
\text { improved clinically }\end{array}$ & $\begin{array}{l}\text { Requires all of the following: } \\
\text { Does not qualify for CR, PR or } \\
\text { progression; stable } \\
\text { nonenhancing (T2/FLAIR) } \\
\text { lesions on the same or lower } \\
\text { dose of corticosteroids } \\
\text { compared with baseline scan. } \\
\text { In the event that the } \\
\text { corticosteroid dose was } \\
\text { increased for new symptoms } \\
\text { and signs without confirmation } \\
\text { of disease progression on } \\
\text { neuroimaging, and subsequent } \\
\text { follow-up imaging shows that } \\
\text { this increase in corticosteroids } \\
\text { was required because of } \\
\text { disease progression, the last } \\
\text { scan considered to show SD will } \\
\text { be the scan obtained when the } \\
\text { corticosteroid dose was } \\
\text { equivalent to the baseline dose }\end{array}$ & $\begin{array}{l}\text { Defined by any of the following: } \\
\geq 25 \% \text { increase in the sum of the } \\
\text { products of perpendicular } \\
\text { diameters of enhancing lesions } \\
\text { compared with the smallest } \\
\text { tumor measurement obtained } \\
\text { either at baseline (if no decrease) } \\
\text { or best response on stable or } \\
\text { increasing doses of } \\
\text { corticosteroids }{ }^{\dagger} \text {; significant } \\
\text { increase in T2/FLAIR } \\
\text { nonenhancing lesion on stable or } \\
\text { increasing doses of } \\
\text { corticosteroids compared with } \\
\text { baseline scan or best response } \\
\text { after initiation of therapy }{ }^{\dagger} \text { not } \\
\text { caused by comorbid events } \\
\text { (e.g., radiation therapy, } \\
\text { demyelination, ischemic injury, } \\
\text { infection, seizures, postoperative } \\
\text { changes or other treatment } \\
\text { effects); any new lesion; clear } \\
\text { clinical deterioration not } \\
\text { attributable to other causes apart } \\
\text { from the tumor (e.g., seizures, } \\
\text { medication adverse effects, } \\
\text { complications of therapy, } \\
\text { cerebrovascular events, infection, } \\
\text { etc.) or changes in corticosteroid } \\
\text { dose; failure to return for } \\
\text { evaluation as a result of death or } \\
\text { deteriorating condition; or clear } \\
\text { progression of nonmeasurable } \\
\text { disease }\end{array}$ \\
\hline
\end{tabular}

$\dagger$ Progression occurs when this criterion is met.

\#Increase in corticosteroids alone will not be taken into account in determining progression in the absence of persistent clinical deterioration.

$\downarrow$ : decrease; $\uparrow$ : increase; CR: Complete response; FLAIR: Fluid-attenuated inversion recovery; HGG: High-grade glioma; NA: Not applicable; PD: Progressive disease; PR: Partial response; SD: Stable disease; T1-Gd +: T1 postgadolinium.

Modified with permission from [4] (C) American Society of Clinical Oncology (2017). All rights reserved.

with bevacizumab, the contrast-enhancing disease may appear stable but there is increase in nonenhancing disease captured on T2/FLAIR sequences indicating disease progression [14]. The MacDonald criteria focus solely on contrast-enhancing disease and does not consider nonenhancing disease progression. The RANO-HGG criteria attempts to address this issue by including increase in nonenhancing disease in the definition of progression (Table 2) [3]. Because of the difficulties in quantifying nonenhancing disease progression, the RANO-HGG criteria suggests that any qualitative increase in nonenhancing disease constitutes progression. This lack of a quantifiable measure of nonenhancing progression is a limitation of the current RANO-HGG criteria. In addition, there is debate regarding the usefulness of evaluating nonenhancing disease in trials involving agents that do not affect vascular permeability. Evaluation of nonenhancing disease is also problematic in immunotherapy trials since these agents are likely to increase peritumoral edema which cannot be easily differentiated from nonenhancing disease progression. As a result this component of the RANO-HGG criteria is often omitted from immunotherapy trials.

The RANO-HGG criteria also attempts to address the phenomenon of pseudoprogression, characterized by an increase in contrast enhancement in the absence of true disease progression, typically following concurrent chemoradiation (Figures $1 \& 2$ ) [15]. Some studies suggest that this occurs more frequently in tumors in which there is methylation of the DNA repair enzyme, O6-methylguanine-DNA methyltransferase (MGMT) [16], although 
(A)

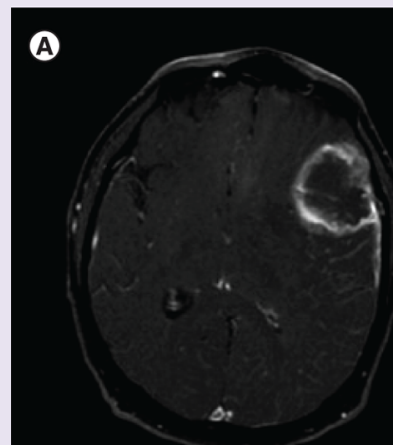

B

$c$

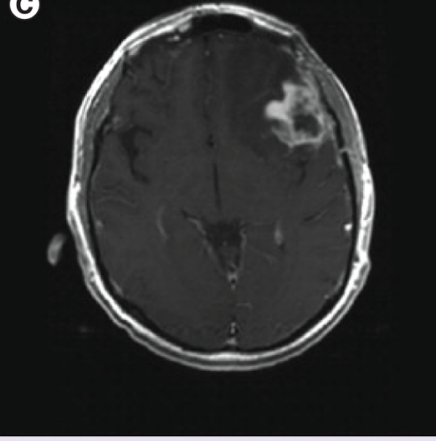

D

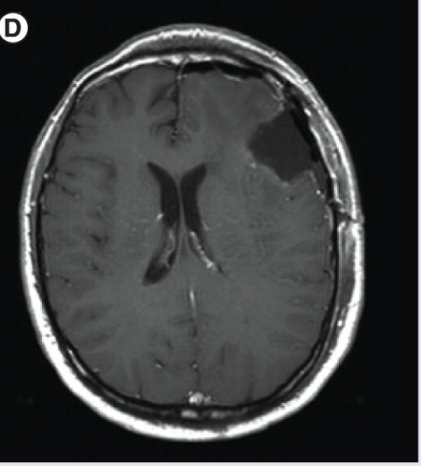

(B)

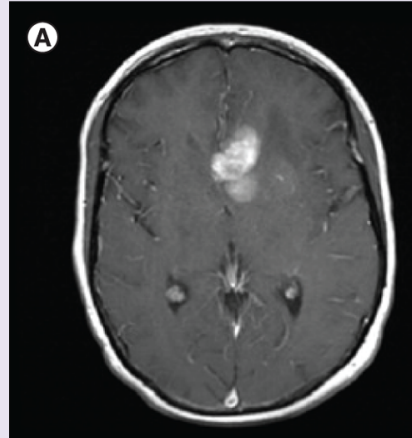

B

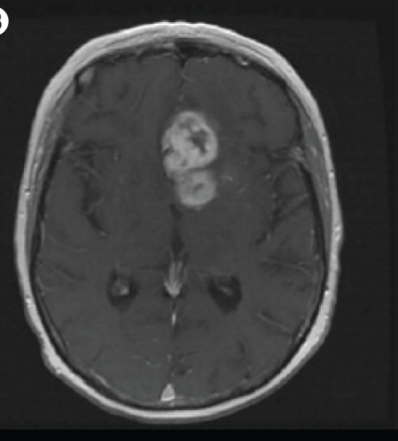

C

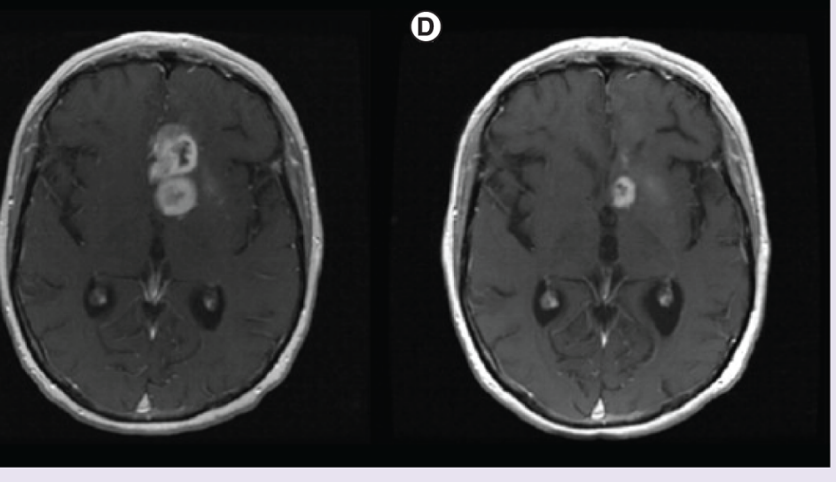

Figure 1. Pseudoprogression after chemoradiotherapy. (A) Axial T1-contrast enhanced MRI (a) before surgery; (b) after surgery; (c) after radiotherapy and concomitant temozolomide showing increased enhancement; (d) re-operation showing only necrotic tissue and no tumor. (B) Pseudoprogression after chemoradiotherapy: axial T1-contrast enhanced MRI showing deep left frontal glioblastoma (a) 2 days after stereotactic biopsy; (b) 4 weeks after radiotherapy and concomitant temozolomide showing increased enhancement, raising the possibility of progression; (c) after four additional weeks of treatment with adjuvant temozolomide showing stable disease; (d) after eight cycles of adjuvant temozolomide showing significant reduction in tumor size. 


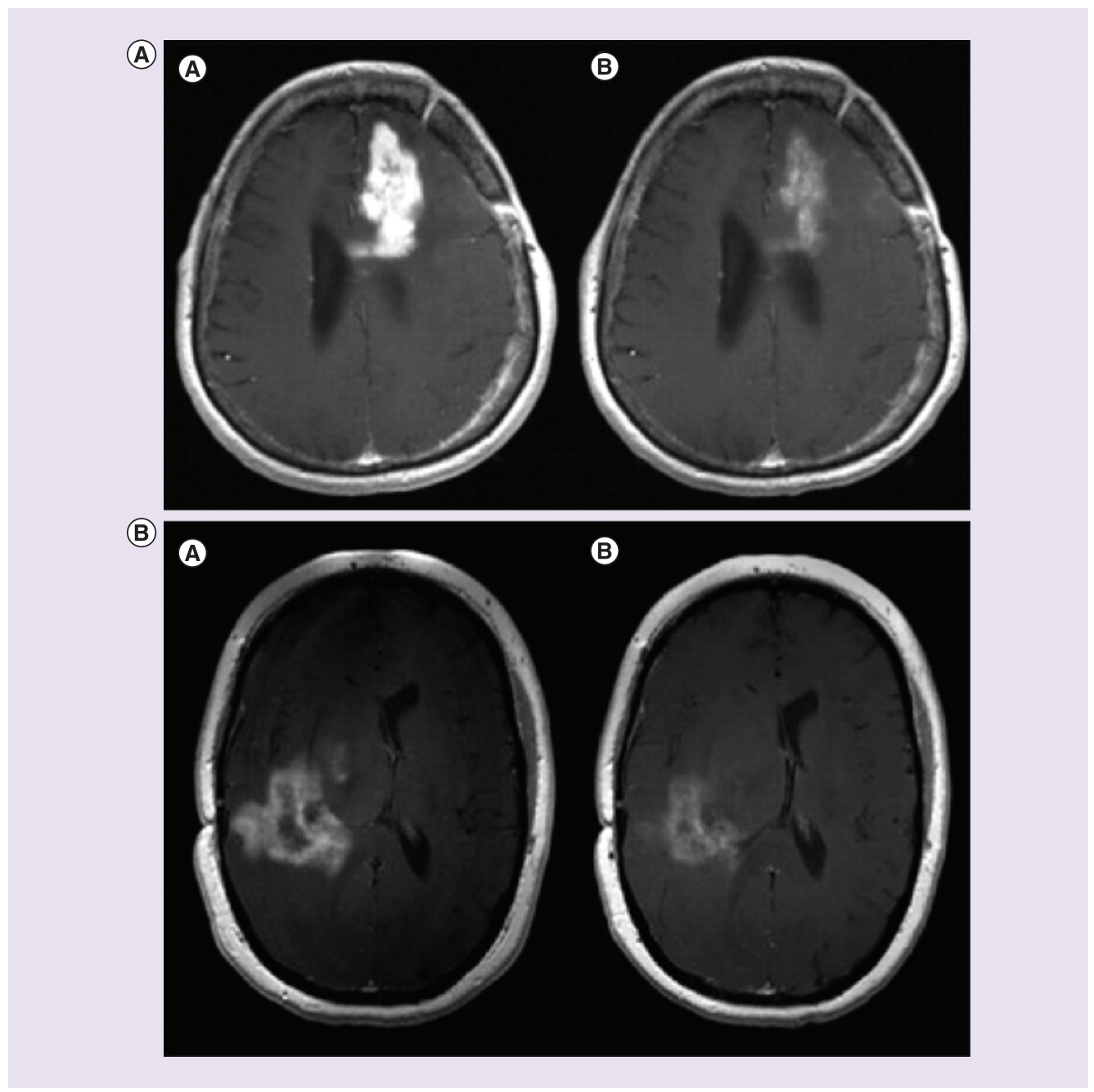

Figure 2. Pseudoresponse. (A) Axial T1-weighted contrast enhanced MRI of left frontal recurrent glioblastoma (a) before and (b) 1 day after therapy with cediranib (pan-VEGFR inhibitor) showing significant reduction in contrast enhancement. The reduction in contrast enhancement within 1 day of therapy is more likely to be caused by reduced vascular permeability to contrast than to a true antitumor effect. (Slide courtesy of A. Gregory Sorensen, Massachusetts General Hospital). (B) Pseudoresponse. Axial T1-weighted contrast enhanced MRI of right parietal glioblastoma (a) before and (b) 1 day after therapy with XL184 (VEGFR and MET inhibitor) showing significant reduction in contrast enhancement. (Slide courtesy of A. Gregory Sorensen, Massachusetts General Hospital).

(A) Adapted with permission from [20].

other studies have not confirmed this [17]. The changes of pseudoprogression are expected to improve with time, whereas true disease progression will continue to increase. In the absence of tissue confirmation, there is no available imaging modality which reliably distinguishes true from pseudoprogression, although perfusion imaging may occasionally be helpful $[18,19]$. The RANO-HGG criteria suggests that within the first 3 months after completion of chemoradiation, even if the MRI shows increase enhancement patients should not be considered to have progressed and be eligible for clinical trials for recurrent disease because of the difficulty in differentiating pseudoprogression from true progression. The exceptions are patients who develop new areas of enhancement outside of the radiation field (beyond the high-dose region or $80 \%$ iso-dose line) or if there is unequivocal evidence of viable tumor on histopathologic sampling. Because of the difficulty in differentiating pseudoprogression from true progression there is the suggestion that the first postradiation MRI, rather than the postoperative MRI, should be used as the baseline scan [18].

The RANO-HGG guidance on pseudoprogression in clinical trials can also be used in routine clinical care of patients. Within the first 3 months after chemoradiation if there is more contrast enhancement within the radiation 
Table 3. Summary of the proposed response assessment in neuro-oncology response criteria for low-grade gliomas.

\begin{tabular}{|c|c|c|c|c|c|}
\hline Criterion & CR & PR & MR & SD & PD \\
\hline T2/FLAIR & $\begin{array}{l}\text { Disappearance of all } \\
\text { lesions }\end{array}$ & $\begin{array}{l}\geq 50 \% \downarrow \text { in perpendicular } \\
\text { diameters of lesion, } \\
\text { sustained for } 4 \text { weeks }\end{array}$ & $\begin{array}{l}25-50 \% \downarrow \text { in } \\
\text { perpendicular diameters } \\
\text { of lesion }\end{array}$ & $<25 \% \downarrow$ to $<25 \% \uparrow$ & $\geq 25 \% \uparrow^{\dagger}$ \\
\hline New lesion & $\begin{array}{l}\text { None (apart from those } \\
\text { consistent with radiation } \\
\text { effects, and no new or } \\
\text { increased enhancement) }\end{array}$ & $\begin{array}{l}\text { None (apart from those } \\
\text { consistent with radiation } \\
\text { effects, and no new or } \\
\text { increased enhancement) }\end{array}$ & $\begin{array}{l}\text { None (apart from those } \\
\text { consistent with radiation } \\
\text { effects, and no new or } \\
\text { increased enhancement) }\end{array}$ & $\begin{array}{l}\text { None (apart from those } \\
\text { consistent with radiation } \\
\text { effects, and no new or } \\
\text { increased enhancement) }\end{array}$ & Present $^{\dagger}$ \\
\hline Corticosteroids & None & Stable or $\downarrow$ & Stable or $\downarrow$ & Stable or $\downarrow$ & $N A^{\ddagger}$ \\
\hline Clinical status & Stable or $\uparrow$ & Stable or $\uparrow$ & Stable or $\uparrow$ & Stable or $\uparrow$ & $\begin{array}{l}\downarrow^{\dagger} \text { (not attributable to } \\
\text { other causes apart from the } \\
\text { tumor, or decrease in } \\
\text { corticosteroid dose) }\end{array}$ \\
\hline Requirement for response & All & All & All & All & Any $\ddagger$ \\
\hline \multicolumn{6}{|c|}{$\begin{array}{l}\text { †Progression occurs when this criterion is met. } \\
\ddagger \text { Increase in corticosteroids alone will not be taken into account in determining progression in the absence of persistent clinical deterioration. } \\
\downarrow \text { : Decrease; } \uparrow: \text { Increase; CR: Complete response; FLAIR: Fluid-attenuated inversion recovery; MR: Minor response; NA: Not applicable; PD: Progressive disease; PR: Partial response; SD: } \\
\text { Stable disease. } \\
\text { Modified with permission from [23] C Elsevier (2011). }\end{array}$} \\
\hline
\end{tabular}

field, but the patient is clinically stable, it is reasonable to continue treatment but monitor the patient closely with serial neuroimaging (e.g., monthly) and consider taking them off treatment only if the subsequent scans confirm progression.

Another useful aspect of RANO-HGG is the guidance it provides for patients with equivocal evidence of progression. The criteria suggest that if these patients are clinically stable they should remain on study and be followed closely with neuroimaging. If subsequent scans confirm progression the date of progression will be backdated to the time when this was first suspected [3]. This approach is similar to the practice often used in routine clinical care.

The guidelines set forth by RANO-HGG also provides guidance in multiple other areas which were not reflected in RECIST or MacDonald criteria. Of particular importance, it provides guidance defining when a patient has adequately progressed to be eligible for enrollment into clinical trials. In general, patients should have a $25 \%$ increase in tumor area to be considered to have progressed. This avoids the inclusion of questionable patients in clinical trials for recurrent disease (Table 2).

\section{RANO-low grade glioma}

Over the past decade, collective understanding of the molecular underpinnings of lower-grade gliomas (LGGs) has increased. This is reflected in the most recent revision of the WHO classification of brain tumors in 2016 [21]. LGGs are now distinguished by both their histological and underlying molecular features, specifically presence or absence of isocitrate dehydrogenase mutations and loss or maintenance of the whole-arm of chromosomes $1 \mathrm{p}$ and $19 \mathrm{q}$ [21]. LGGs have been considered historically to be slower growing and radiographically distinct from high-grade gliomas, and characterized by minimal, if any, evidence of contrast enhancement and nonspecific, T2/FLAIR hyperintense lesions with often indistinct margins. In the appropriate clinical context, MRI findings in LGG may be confused with other processes including demyelination, ischemia or late effects of cranial irradiation [3,6].

The RANO criteria for LGG utilizes percent change in T2/FLAIR signal rather than contrast enhancement for determination of response and progression (unlike RANO-HGG) [22]. In addition, since responses ( $>50 \%$ decrease in area) are relatively uncommon with most agents tested to date, the RANO LGG criteria introduced a minor response category ( $>25 \%$ but $<50 \%$ decrease in area; Table 3). As with RANO-HGG, corticosteroid use and clinical status are considered in the determination of response and progression.

One of the challenges in determining response and progression in LGGs is the difficulty in accurately measuring the tumor using only 2D. There is ongoing work to determine if measuring T2/FLAIR volume is more accurate in determining changes in tumor burden and whether newer approaches such as determining changes in tumor volume growth trajectory can be a more reliable and sensitive measure of response [24].

In addition to determining changes in tumor size, other measures of clinical benefit such as changes in neurocognitive function, quality of life and seizures may be useful in assessing the utility of novel therapies. In particular, 
Table 4. Summary of the proposed response assessment in neuro-oncology response criteria for brain metastases.

\begin{tabular}{|c|c|c|c|c|}
\hline Criterion & CR & PR & SD & PD \\
\hline Target lesions & None & $\begin{array}{l}\geq 30 \% \text { decrease in sum LD } \\
\text { relative to baseline }\end{array}$ & $\begin{array}{l}<30 \% \text { decrease relative to } \\
\text { baseline, but }<20 \% \text { increase in } \\
\text { sum LD relative to nadir }\end{array}$ & $\begin{array}{l}\geq 20 \% \text { increase in sum LD relative } \\
\text { to } \text { nadir }^{\dagger}\end{array}$ \\
\hline Nontarget lesions & None & Stable or improved & Stable or improved & Unequivocal PD ${ }^{\dagger}$ \\
\hline New lesion(s) $)^{\ddagger}$ & None & None & None & Present $^{\dagger}$ \\
\hline Corticosteroids & None & Stable or decreased & Stable or decreased & $N A^{\S}$ \\
\hline Clinical status & Stable or improved & Stable or improved & Stable or improved & Worse $^{\dagger}$ \\
\hline Requirement for response & All & All & All & Any ${ }^{\S}$ \\
\hline \multicolumn{5}{|c|}{$\begin{array}{l}\text { †Progression occurs when this criterion is met. } \\
¥ \text { New lesion = new lesion not present on prior scans and visible in at least two projections. If a new lesion is equivocal, for example, because of its small size, continued therapy may } \\
\text { considered and follow-up evaluation will clarify whether it represents truly new disease. If repeat scans confirm there is definitely a new lesion, then progression should be declared usir } \\
\text { the date of the initial scan showing the new lesion. For immunotherapy-based approaches, new lesions alone do not define progression. } \\
\S \text { Increase in corticosteroids alone will not be taken into account in determining progression in the absence of persistent clinical deterioration. } \\
\text { CR: Complete response; LD: Longest dimension; NA: Not applicable; PD: Progressive disease; PR: Partial response; SD: Stable disease. } \\
\text { Reprinted with permission from [6] (C) Elsevier (2015). }\end{array}$} \\
\hline
\end{tabular}

seizure control has recently been proposed as an additional metric in disease response and as an endpoint in trials for LGGs [25].

\section{RANO-brain metastases}

CNS metastases represent an important and disabling neurologic complication of systemic cancer, occurring in up to $40 \%$ of patients with metastatic cancer [26]. Once BM is developed, management typically entails local therapies, such as surgical resection or radiation, whereas several driver mutations have been identified in systemic malignancies, resulting in development of targeted drugs, notably in non-small-cell lung cancer, melanoma and breast cancers, the reach of these agents on associated BM has been modest, attributable to multiple factors including access of agents cross the blood-brain and blood-tumor barrier, and tumor heterogeneity) [26]. There are also practical considerations, in that patients with BM, specifically symptomatic lesions, have historically been excluded from clinical trials in which these agents have been investigated [27-31]. This has resulted in limited understanding of efficacy of agents in this population and in determining reliable endpoints for how disease response can be appropriately obtained.

Prior to establishment of the RANO-BM working group, clinical trials in systemic cancers often used the RECIST criteria for response assessment and had limitations when applied to BM, including the need to summate both CNS and systemic lesions as they were considered a single compartment [32,33]. There were other shortcomings in the approach to enrolling BM patients on to trial, given the inherent heterogeneity of histology and patient population, and provider preferences with regards to imaging and frequency of monitoring [32]. As trials may have incorporated any of the response assessment criteria available, this led to lack of uniformity in comparing effects of treatments across studies [32]. The RANO-BM working group first worked to propose standard criteria for determining response and progression in BM trials based on consensus opinion [34]. These response criteria were based upon factors derived from RANO-HGG as well as RECIST. Since BM are generally spherical, 1D measurement was used. As with RECIST, progression occurs when the sum of the linear measurements exceeds $20 \%$ compared with baseline or best response and response is defined as reduction of the sum of linear measurements by 30\% compared with baseline (Table 4) [34]. As with RANO-HGG, the patient's clinical status and corticosteroid use are considered. Unlike RECIST, the brain is considered a separate compartment, allowing the status of disease systemically and in the brain to be considered separately, if appropriate. RANO-BM defines measurable disease as lesions of $1 \mathrm{~cm}$ or larger, although there is guidance if smaller lesions are allowed [34]. In addition, it provides guidance on the number of target lesions, use of corticosteroids and issues related to pseudoprogression from radiosurgery and immunotherapy [34]. As with RANO-HGG, these criteria are primarily for determining response in patients enrolled in clinical trials but could also be applied to patients receiving routine clinical care.

As designing appropriate clinical trials for patients with BM remains challenging, RANO BM has also established separate guidelines for clinical trials in which either local or systemic treatments are investigated [35,36].

The rationale for local therapy, specifically surgery and radiation therapy (radiosurgery and whole-brain radiation), is delay intracranial progression and improvement in local disease control. The RANO-BM working 
group, in consideration of clinical trials in which local treatments are investigated, proposes different endpoints and guidance in determining endpoints, depending on the planned intervention and design and phase of the trial. Early-phase trials are likely to emphasize improvement in intracranial control while later phase may prioritize, toxicity, functional and patient-reported outcomes (PROs) [35]. The use of advanced imaging modalities is also recommended by RANO-BM, specifically in assessing radiographic changes which may reflect the effect of treatment, causing pseudoprogression. MR perfusion, MR spectroscopy and positron emission tomography (PET) imaging are suggested techniques which may sometimes be useful in distinguishing true disease from pseudoprogression, if available [35].

In aiming to standardize and improve clinical trials in which systemic agents are investigated in patients with $\mathrm{BM}$, the RANO-BM offers the following: strategies to determine potential CNS activity of the drug in question, understanding the potential disease response both intra- and extracranially, the potential for prevention of BM in high-risk disease patients and last, relevant endpoints [35]. As it is expected that the incidence of patients living with controlled systemic malignancy will rise, strategies for controlling or preventing BM will become increasingly important.

\section{Immunotherapy RANO}

There has been increasing use of the immunotherapies in management of systemic cancers, and a rapidly growing number of approvals by US FDA [37]. Immunotherapy, including vaccines, oncolytic viruses and programmed death (PD), programmed death ligand 1 (PD-L1) and cytotoxic T-lymphocyte 4 (CTLA-4) antibodies, are being investigated in primary and metastatic brain tumors. Although there is significant enthusiasm around use of these agents, understanding radiographic changes associated with their use has presented challenges [38]. Drawing from the experiences in solid tumor oncology, especially melanoma, true radiographic response is sometimes preceded by transient worsening $[39,40]$.

The immunotherapy RANO (iRANO) working group has sought to establish guidelines for determining response with the aim of preventing premature assignment of patients as nonresponders. The criteria are similar to what has been established by RANO-HGG, LGG and BM, using complete response, partial response, stable disease and $\mathrm{PD}$ as endpoints. The additional guidance provided by iRANO is that within the first 6 months of treatment with an immunotherapy, if the scans are worse, but if the patient is clinically stable, they can stay on treatment and be closely observed with serial imagers over the next 3 months. Only if subsequent scans confirm progression should they be taken off the study medication. These recommendations will require validation. Nonetheless they provide useful guidance on the management of patients receiving immunotherapies, not only on clinical trials, but also receiving routine clinical care (Figure 3).

\section{Neurological assessment in neuro-oncology}

Although both the MacDonald and RANO criteria include clinical status in the determination of progression, there is no quantifiable measure to determine this. The neurological assessment in neuro-oncology (NANO) was created to address this limitation and quantify clinical outcomes [3]. The NANO working group has put forth a disease-specific clinical assessment scale, using parts of routine neurologic examination to measure neurologic function across multiple domains [41]. The scale may be conducted and reported by a trained healthcare professional, testing patients in the following: gait, strength, sensation, visual field, facial strength, language, behavior and upper extremity ataxia, with scores in each domain ranging from 0 to 3 or 0 to 2 [41]. In pilot efforts, NANO has been incorporated into clinical trials and has been associated with high interobserver agreement and reliability. Although not intended to replace current standard neurologic assessments in the clinic, NANO is an objective and simple measure which can be integrated into clinical trials for neuro-oncology patients, thus potentially providing a more objective tool for determination of disease progression. The ultimate role of the NANO scale will depend on ongoing efforts to validate this instrument in clinical trials.

\section{RANO leptomeningeal metastases}

Leptomeningeal disease (LMD) is a feared and late neurologic complication of systemic cancer, most commonly occurring in solid tumors including breast cancer and lung cancer as well as hematologic malignancies [42,43]. Primary brain tumors may also disseminate the cerebrospinal fluid (CSF) or leptomeninges, especially medulloblastoma. To date, few effective systemic therapies have been identified and management recommendations often includes radiation therapy, administration of chemotherapy into the CSF or systemic therapy. 


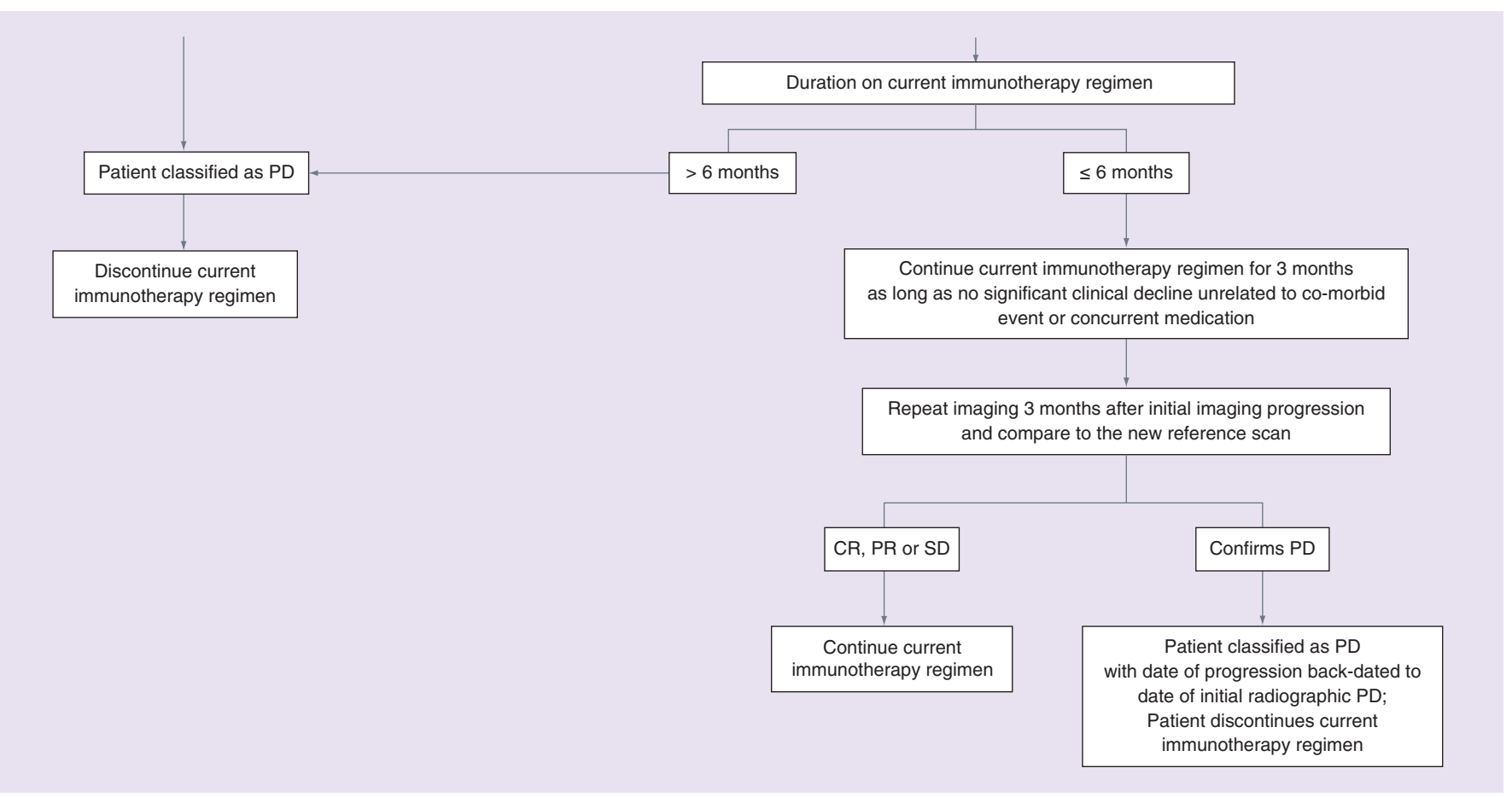

Figure 3. Response Assessment in Neuro-Oncology treatment algorithm for the assessment of progressive imaging findings in neuro-oncology patients treated with immunotherapy.

CR: Complete response; PD: Progressive disease; PR: Partial response; SD: Stable disease.

The RANO-LM working group recently proposed criteria to quantify the extent of disease and guide the determination of response and progression [20]. These criteria include a neurologic scale, like NANO [3,20,42] together with neuroimaging (brain and spine) and CSF cytology, which would be required at each assessment. Although these criteria incorporate the components that contribute to the routine clinical evaluation of LM patients, these have yet to be validated [20]. For now, the complexity of the RANO-LM scale probably limits its utility for routine clinical care outside of clinical trials. There is additionally, a parallel effort by the European Association of Neuro-Oncology (EANO) and European Society of Medical Oncology to put forth guidelines for diagnosis, monitoring and management of LMD from solid tumors, with levels of evidence to support their recommendations. It is noted that the EANO/European Society of Medical Oncology practice guidelines does not cover LMD from hematologic malignancies or primary brain tumors [44].

\section{RANO-corticosteroids}

Corticosteroids, in varying doses, are invariably an integral part of clinical management of patients with CNS tumors at some point in their care, providing symptom control and preserving function. The side effects associated with prolonged use of corticosteroids are well-characterized and far-reaching. It has also been proposed that patients requiring steroids for longer periods of time or at higher doses have shorter overall survival. Additionally, steroid use, along with age and performance status, has been associated with poorer prognosis [45,46]. Therapies such as bevacizumab are known to aid in decreasing steroid requirement and sparing associated side effects; historically, clinical trials have used in steroid dose (or time to steroid initiation) as secondary endpoints [47]. Previous attempts in investigating alternate agents for steroid-sparing properties have been promising, specifically the agent corticorelin, which had been studied in a Phase I in pediatric patients in treatment of peritumoral edema. Early results included improvement in steroid dose reduction and quality-of-life indices; however, further investigation was not pursued by the study sponsors [48].

A working group for development of consensus criteria for evaluation of therapeutic response in the setting of corticosteroid has been established, borne out of the need for consistent guidelines in determining relevant clinical and radiographic endpoints, specifically in consideration of the impact of steroids on disease. To this end, criteria for response while on corticosteroids have been proposed for both adult and pediatric patients [49]. The criteria 
Table 5. Side effects of corticosteroids.

\begin{tabular}{|c|c|}
\hline Organ system & Adverse effect \\
\hline \multirow[t]{6}{*}{ Neurological/Psychiatric } & Insomnia \\
\hline & Mood lability \\
\hline & Anxiety/depression \\
\hline & Psychosis \\
\hline & Increased appetite \\
\hline & Hiccups \\
\hline \multirow{4}{*}{ Musculoskeletal } & Osteoporosis \\
\hline & Arthralgias \\
\hline & Avascular necrosis \\
\hline & Decreased growth/height (pediatric patients) \\
\hline Gastrointestinal & Dyspepsia/gastritis \\
\hline \multirow{3}{*}{ Endocrine } & Weight gain \\
\hline & Cushingoid habitus \\
\hline & Adrenal insufficiency (after discontinuation) \\
\hline \multirow[t]{5}{*}{ Cutaneous or Vascular } & Acne \\
\hline & Striae \\
\hline & Purpura \\
\hline & Delayed wound healing \\
\hline & Peripheral edema \\
\hline \multirow[t]{2}{*}{ Ocular } & Visual blurring \\
\hline & Cataract formation \\
\hline
\end{tabular}

incorporate dose changes, improvement in neurologic and/or functional status and duration of benefit (Table 5). Additional criteria proposed for adult patients is based upon improved score on PROs, with specific preference made for the dexamethasone symptom questionnaire-chronic, though this is limited by the absence of defined meaningful change [49]. This RANO proposal aimed at designing endpoints for corticosteroid use in clinical trials may need to be defined and will require prospective validation in clinical studies.

\section{RANO-positron emission tomography}

MRI has been the standard imaging modality for evaluation of disease activity and treatment response in highgrade glioma. Although there are advantages to continued use of contrast-enhanced imaging in assessing treatment response, there remains multiple pitfalls, particularly in the advent of anti-angiogenic and immune checkpoint blockade, thus making interpretation challenging. Although this forms the rationale for development of RANO working groups including iRANO, there is also impetus to explore alternate, advanced imaging modalities, namely, PET [50]. Specific PET modalities including glucose-based ${ }^{18} \mathrm{~F}$-fluorodeoxy-glucose $\left({ }^{18} \mathrm{~F}-\mathrm{FDG}\right)$ and amino acidbased, specifically ${ }^{11} \mathrm{C}$-methyl-methionine $\left({ }^{11} \mathrm{C}\right.$-MET $), 18 \mathrm{~F}$-fluorophenylalanine $\left({ }^{18} \mathrm{~F}\right.$-DOPA $)$ and ${ }^{18} \mathrm{~F}$-fluoroethylL-tyrosine $\left({ }^{18} \mathrm{~F}\right.$-FET), have been studied as complimentary to MR-based imaging to evaluate disease response [51].

To this end, the collaboration of RANO with the EANO has put forth guidelines for clinical management patients with glioma. The recommendations provided are relevant to specific milestones in management, from incorporation of specific PET modalities in diagnosis to informing discussion around treatment response and disease prognostication [50]. Potentially challenging broader use of are the limited availability of centers which offer both glucose and amino acid-based PET. Formal guidelines by RANO/EANO are pending.

There is a separate effort by RANO to develop evidence-based recommendations for use of PET imaging in management of patients with meningioma [52]. There are challenges to widespread adoption of PET in meningioma, including determining where in management is incorporation of its use most relevant. Currently, the RANO-PET recommendations for meningioma are aimed at informing daily management, thus serving as a guideline for 
clinicians. With further validation in larger cohorts, this may be expanded to determine endpoints for relevant clinical trials.

\section{Response assessment in pediatric neuro-oncology}

Consistent with the aims of RANO, the response assessment in pediatric neuro-oncology (RAPNO) was established to address limitations in determining treatment response, specifically in pediatric brain tumors. Although there are concerns which are shared by both adult and pediatric groups, there are separate challenges, unique to pediatric neuro-oncology, which warrant different consideration as it relates to assessment of disease response [53]. RAPNO has been tasked with determining relevant consensus criteria for assessing treatment response; however, across multiple and heterogeneous tumor types, each representing a distinct disease with variable imaging features [54]. Currently, no standard guidelines exist for evaluating pediatric brain tumor treatment response and the adult have formed the basis for assessment to date [54].

Tumor-specific guidelines are also in development through RAPNO for establishing criteria in high-grade glioma, optic pathway glioma, diffuse midline glioma and leptomeningeal disease, with recent recommendations made for medulloblastoma and similar tumors with propensity for CSF dissemination. Currently, there are no consensus criteria for determining response in clinical trials in both adult and pediatric patients with this disease. RAPNO has put forth initial recommendations for response criteria, which is based upon methods including CNS MR findings, CSF cytology and neurologic examination. Other additional criteria were considered, such as presence of extra-CNS metastatic disease and quality of life factors; however, no specific recommendations have yet been made to include in the RAPNO criteria [55]. This RANO proposal will require prospective validation in clinical studies.

\section{Other RANO working groups}

Other working groups in RANO have been formed to establish guidelines for use in clinical trials, including individual efforts focused upon surgery, spinal metastatic disease, meningioma and PROs.

\section{Conclusion \& future perspective}

The RANO working groups have proposed to establish standard and reliable criteria for objective assessment of treatment response. Given the advances in understanding of molecular underpinnings of primary and metastatic brain tumors, there will be opportunities to exploit these changes for therapeutic benefit and rigorous investigation of potential agents will be necessary. As this is a dynamic process, it is expected that in the future, as more data accumulates, guidelines proposed by the RANO working groups will reflect and will be informed by newly gathered information.

\section{Executive summary}

Response assessment in neuro-oncology-high grade glioma

- There have been multiple challenges to development of effective treatments for high-grade glioma including lack of molecular targets, tumor heterogeneity, small patient population and blood-brain barrier considerations.

- Response assessment in neuro-oncology-high grade glioma (RANO-HGG) was the first of the RANO working groups to be established, tasked with developing and determining important and accepted endpoints for clinical trials.

- Building upon the experience from Response Evaluation Criteria in Solid Tumors and MacDonald criteria, RANO-HGG addresses enhancing and nonenhancing disease, use of corticosteroids and provides guidance around defining true radiographic versus 'pseudoprogression,' which has become more prominent in the era of anti-angiogenic and other agents which disturb vascular permeability.

\section{RANO-low grade glioma}

- Low-grade gliomas (LGGs) are distinguished by both their histological and underlying molecular features, which has been captured in the 2016 WHO classification of brain tumors.

- Imaging findings in LGGs are often similar to other processes such as demyelination or ischemia; additionally, the slow growth pattern which is characteristic of these tumors often render assessing disease response challenging.

- RANO-LGG has put forth guidelines, relying upon changes to T2/FLAIR signal (in place of contrast enhancement), as well as clinical status and corticosteroid dose change similar to RANO-HGG, as part of criteria for disease response and progression.

RANO-brain metastases

- Brain metastases are a frequent complication of systemic cancer, occurring most frequently in melanoma, breast and lung cancers. 
- Management has been limited to local therapies such as surgery and radiation. Despite improved understanding and increased use of targeted therapies in systemic cancers, the therapeutic benefits of these agents have been modest in treatment of brain metastases.

- The goals of RANO-BM are to determine CNS activity of potential agents and to determine relevant endpoints for clinical trials.

Immunotherapy RANO

- Immunotherapies have been increasing in use in systemic malignancies with several agents having recently gained approval for standard-of-care use.

- These agents are now also being investigated in management of both primary and metastatic brain tumors.

- Immunotherapy RANO was established to determine guidelines for response in patients treated with immunotherapy.

- Similar to efforts in solid tumor oncology, immunotherapy RANO aims to identify and determine criteria to reduce premature declaration of true progression, as true radiographic response is often preceded by transient worsening.

Neurological assessment in neuro-oncology

- Neurological assessment in neuro-oncology (NANO) was created to provide a more objective tool for determination of disease progression.

- It is an objective and simple measure which can be integrated into clinical trials for neuro-oncology patients.

RANO-leptomeningeal metastases

- Leptomeningeal metastases (LM) is a devastating complication of systemic complication with few available systemic treatment options.

- Criteria for quantifying disease progression and response in LM has been recently proposed and its use in routine clinical settings will need to be determined.

RANO corticosteroids

- Corticosteroids, though an integral part of symptom management of patients with CNS tumors, are associated with significant side effects and potential for shorter overall survival.

- Given the impact of corticosteroids on disease, the RANO working group is tasked with designing endpoints for corticosteroid use in clinical trials.

RANO-positron emission tomography

- Positron emission tomography may be a compliment to MR-based imaging in management of patients with high-grade glioma and meningioma.

- There is a collaborative effort with RANO and European Association of Neuro-Oncology to develop evidence-based guidelines for use of positron emission tomography in standard management and ultimately, clinical trials.

Response assessment in pediatric neuro-oncology

- There are unique challenges to determining treatment response in pediatric brain tumors, thus warranting separate consideration.

- Initial recommendations have been put forth for management of medulloblastoma and cerebrospinal fluid-seeding tumors.

- response assessment in pediatric neuro-oncology is developing tumor-specific guidelines for pediatric brain tumors including high-grade glioma, optic pathway glioma and diffuse midline glioma.

Other RANO working groups

- Efforts to guidelines for disease response have been initiated in multiple additional areas including on corticosteroid use, surgery, spinal metastatic disease, meningioma and patient-reported outcomes.

Conclusion \& future perspective

- RANO working groups have been established to determine relevant and reliable criteria for assessing disease response.

\section{Financial \& competing interests disclosure}

The authors have no relevant affiliations or financial involvement with any organization or entity with a financial interest in or financial conflict with the subject matter or materials discussed in the manuscript. This includes employment, consultancies, honoraria, stock ownership or options, expert testimony, grants or patents received or pending, or royalties.

No writing assistance was utilized in the production of this manuscript. 


\section{References}

Papers of special note have been highlighted as: $\bullet \bullet$ of considerable interest

1. Ostrom QT, Gittleman H, Liao P et al. CBTRUS statistical report: primary brain and other central nervous system tumors diagnosed in the United States in 2010-2014. Neuro Oncol. 19(Suppl. 5), v1-v88 (2017) doi: 10.1093/neuonc/nox158.

2. Chukwueke UN, Brastianos PK. Sequencing brain metastases and opportunities for targeted therapies. Pharmacogenomics 18(6), 585-594 (2017) doi: 10.2217/pgs-2016-0170.

3. Wen PY, Chang SM, Van den Bent MJ et al. Response assessment in neuro-oncology clinical trials. J. Clin. Oncol. 35(21), 2439-2449 (2017) doi:10.1200/JCO.2017.72.7511.

-. Provides an overview of many of the Response Assessment in Neuro-Oncology (RANO) working groups and summarizes their efforts.

4. Levin VA, Crafts DC, Norman DM et al. Criteria for evaluating patients undergoing chemotherapy for malignant brain tumors. J. Neurosurg. 47(3), 329-335 (1977).

5. MacDonald DR, Cascino TL, Schold SC Jr et al. Response criteria for Phase II studies of supratentorial malignant glioma. J. Clin. Oncol. 8(7), 1277-1280 (1990).

6. Eisele SC, Wen PY, Lee EQ. Assessment of brain tumor response: RANO and its offspring. Curr. Treat. Options Oncol. 17(7), 35 (2016) doi: 10.1007/s11864-016-0413-5.

7. Stupp R, Mason WP, van den Bent MJ et al. Radiotherapy plus concomitant and adjuvant temozolomide for glioblastoma. N. Engl. J. Med. 352(10), 987-996 (2005).

8. Taphoorn MJB, Dirven L, Kanner AA et al. Influence of treatment with tumor-treating fields on health-related quality of life of patients with newly diagnosed glioblastoma: a secondary analysis of a randomized clinical trial. JAMA Oncol. (2018) doi: 10.1001/jamaoncol.2017.5082 (Epub ahead of print).

9. Stupp R, Taillibert S, Kanner AA et al. Maintenance therapy with tumor-treating fields plus temozolomide vs temozolomide alone for glioblastoma: a randomized clinical trial. JAMA 314(23), 2535-2543 (2015) doi: 10.1001/jama.2015.16669.

10. Alexander BM, Lee EQ, Reardon DA, Wen PY. Current and future directions for Phase II trials in high-grade glioma. Expert Rev. Neurother. 13(4), 369-387 (2013) doi: 10.1586/ern.12.158.

11. Wen PY, Cloughesy TF, Ellingson BM et al. Report of the jumpstarting brain tumor drug development coalition and FDA clinical trials neuroimaging endpoint workshop (January 30, 2014, Bethesda MD). Neuro. Oncol. 16 (Suppl. 7), vii36-vii47 (2014) doi:10.1093/neuonc/nou226.

12. Gilbert MR, Dignam JJ, Armstrong TS et al. A randomized trial of bevacizumab for newly diagnosed glioblastoma. $N$. Engl. J. Med. 370(8), 699-708 (2014) doi: 10.1056/NEJMoa1308573.

13. van den Bent MJ, Vogelbaum MA, Wen PY, MacDonald DR, Chang SM. End point assessment in gliomas: novel treatments limit usefulness of classical MacDonald's Criteria. J. Clin. Oncol. 27(18), 2905-2908 (2009) doi: 10.1200/JCO.2009.22.4998.

-• Provides historical context for the development and rationale for RANO.

14. Delgado-López PD, Riñones-Mena E, Corrales-García EM. Treatment-related changes in glioblastoma: a review on the controversies in response assessment criteria and the concepts of true progression, pseudoprogression, pseudoresponse and radionecrosis. Clin. Transl. Oncol. (2017) doi: 10.1007/s12094-017-1816-x (Epub ahead of print).

15. Gállego Pérez-Larraya J, Lahutte M, Petrirena G et al. Response assessment in recurrent glioblastoma treated with irinotecan-bevacizumab: comparative analysis of the MacDonald, RECIST, RANO, and RECIST + F criteria. Neuro Oncol. 14(5), 667-673 (2012) doi: 10.1093/neuonc/nos070.

-• Summarizes the current response criteria in neuro-oncology.

16. Reardon DA, Ballman KV, Buckner JC, Chang SM, Ellingson BM. Impact of imaging measurements on response assessment in glioblastoma clinical trials. Neuro Oncol. 16 (Suppl. 7), vii24-vii35 (2014) doi: 10.1093/neuonc/nou286.

17. Chakravarti A, Erkkinen MG, Nestler U et al. Temozolomide-mediated radiation enhancement in glioblastoma: a report on underlying mechanisms. Clin. Cancer Res. 12(15), 4738-4746 (2006).

18. Wick W, Chinot OL, Bendszus M et al. Evaluation of pseudoprogression rates and tumor progression patterns in a Phase III trial of bevacizumab plus radiotherapy/temozolomide for newly diagnosed glioblastoma. Neuro Oncol. 18(10), 1434-1441 (2016) doi: 10.1093/neuonc/now091.

19. Ellingson BM, Wen PY, Cloughesy TF. Modified criteria for radiographic response assessment in glioblastoma clinical trials. Neurotherapeutics 14(2), 307-320 (2017) doi: 10.1007/s13311-016-0507-6.

-. Provides an excellent overview of the strengths and pitfalls of the current RANO criteria with recommendations made for potential solutions.

20. Chamberlain M, Junck L, Brandsma D et al. Leptomeningeal metastases: a RANO proposal for response criteria. Neuro Oncol. 19(4), 484-492 (2017) doi: 10.1093/neuonc/now183.

21. Wen PY, MacDonald DR, Reardon DA et al. Updated response assessment criteria for high-grade gliomas: response assessment in neuro-oncology working group. J. Clin. Oncol. 28(11), 1963-1972 (2010) doi: 10.1200/JCO.2009.26.3541. 
22. Louis DN, Perry A, Reifenberger G et al. The 2016 World Health Organization Classification of tumors of the central nervous system: a summary. Acta Neuropathol. 131(6), 803-820 (2016) doi: 10.1007/s00401-016-1545-1.

23. Henson JW, Ulmer S, Harris GJ. Brain tumor imaging in clinical trials. AJNR Am. J. Neuroradiol. 29(3), 419-24 (2008) doi: 10.3174/ajnr.A0963.

-. Discusses the evolution of use of various imaging and response criteria in clinical trials.

24. van den Bent MJ, Smits M, Kros JM et al. Diffuse infiltrating oligodendroglioma and astrocytoma. J. Clin. Oncol. 35(21), 2394-2401 (2017) doi: 10.1200/JCO.2017.72.6737.

25. Huang RY, Young R, Ellingson B et al. Volumetric analysis of IDH-mutant low-grade glioma: a natural history study of tumor growth rates before and after treatment. In: Society of Neuro-Oncology (2017) CA and TX, USA, S No. 2017, Abstract No. 50.

26. Avila EK, Chamberlain M, Schiff $\mathrm{D}$ et al. Seizure control as a new metric in assessing efficacy of tumor treatment in low-grade glioma trials. Neuro Oncol. 19(1), 12-21 (2017) doi: 10.1093/neuonc/now190.

27. Berghoff AS, Brastianos PK. Toward precision medicine in brain metastases. Semin. Neurol. 38(1), 95-103 (2018) doi: $10.1055 / \mathrm{s}-0038-1627469$.

28. McCoach CE, Berge EM, Lu X, Barón AE, Camidge DR. A brief report of the status of central nervous system metastasis enrollment criteria for advanced non-small cell lung cancer clinical trials: a review of the ClinicalTrials.gov trial registry. J. Thorac. Oncol. 11(3), 407-413 (2016) doi: 10.1016/j.jtho.2015.10.024.

29. Borghaei H, Paz-Ares L, Horn L et al. Nivolumab versus docetaxel in advanced nonsquamous non-small-cell lung cancer. N. Engl. J. Med. 373(17), 1627-1639 (2015) doi: 10.1056/NEJMoa1507643.

30. Robert C, Long GV, Brady B et al. Nivolumab in previously untreated melanoma without BRAF mutation. N. Engl. J. Med. 372(4), 320-330 (2015) doi: 10.1056/NEJMoa1412082.

31. Brastianos PK, Curry WT, Oh KS. Clinical discussion and review of the management of brain metastases. J. Natl Compr. Canc. Netw. 11(9), 1153-1164 (2013).

32. Arvold ND, Lee EQ, Mehta MP et al. Updates in the management of brain metastases. Neuro Oncol. 18(8), 1043-1065 (2016) doi: $10.1093 /$ neuonc/now127.

33. Lin NU, Lee EQ, Aoyama $\mathrm{H}$ et al. Challenges relating to solid tumour brain metastases in clinical trials, part 1: patient population, response, and progression. A report from the RANO group. Lancet Oncol. 14, e396-e406 (2013).

34. Quant EC, Wen PY. Response assessment in neuro-oncology. Curr. Oncol. Rep. 13(1), 50-56 (2011) doi: 10.1007/s11912-010-0143-y.

35. Lin NU, Lee EQ, Aoyama $\mathrm{H}$ et al. Response assessment criteria for brain metastases: proposal from the RANO group. Lancet Oncol. 16(6), e270-e278 (2015) doi: 10.1016/S1470-2045(15)70057-4.

36. Alexander BM, Brown PD, Ahluwalia MS et al. Clinical trial design for local therapies for brain metastases: a guideline by the Response Assessment in Neuro-Oncology Brain Metastases working group. Lancet Oncol. 19(1), e33-e42 (2018) doi: 10.1016/S1470-2045(17)30692-7.

37. Camidge DR, Lee EQ, Lin NU et al. Clinical trial design for systemic agents in patients with brain metastases from solid tumours: a guideline by the Response Assessment in Neuro-Oncology Brain Metastases working group. Lancet Oncol. 19(1), e20-e32 (2018) doi: 10.1016/S1470-2045(17)30693-9.

38. Okada H, Weller M, Huang R et al. Immunotherapy response assessment in neuro-oncology: a report of the RANO working group. Lancet Oncol. 16(15), e534-e542 (2015) doi: 10.1016/S1470-2045(15)00088-1.

39. Hodi FS, O’Day SJ, McDermott DF et al. Improved survival with ipilimumab in patients with metastatic melanoma. $N$. Engl. J. Med. 363(8), 711-723 (2010) doi: 10.1056/NEJMoa1003466.

40. Hamid O, Robert C, Daud A et al. Safety and tumor responses with lambrolizumab (anti-PD-1) in melanoma. N. Engl. J. Med. 369(2), 134-144 (2013) doi: 10.1056/NEJMoa1305133.

41. Wolchok JD, Hoos A, O'Day S et al. Guidelines for the evaluation of immune therapy activity in solid tumors: immune-related response criteria. Clin. Cancer Res. 15(23), 7412-7420 (2009) doi: 10.1158/1078-0432.CCR-09-1624.

-• Summarizes the current efforts and response criteria for assessing solid tumors following treatment with immuno-oncologic agents.

42. Nayak L, DeAngelis LM, Brandes AA et al. Neurologic Assessment in Neuro-Oncology (NANO) scale: a tool to assess neurologic function for integration into the Response Assessment in Neuro-Oncology (RANO) criteria. Neuro Oncol. 19(5), 625-635 (2017) doi: $10.1093 /$ neuonc/nox029.

43. Kesari S, Batchelor TT. Leptomeningeal metastases. Neurol. Clin. 21(1), 25-66 (2003).

44. Le Rhun E, Weller M, Brandsma D et al. EANO-ESMO clinical practice guidelines for diagnosis, treatment and follow-up of patients with leptomeningeal metastasis from solid tumours. Ann Oncol. 28(Suppl. 4), iv84-iv99 (2017).

45. Michaelsen SR, Christensen IJ, Grunnet $\mathrm{K}$ et al. Clinical variables serve as prognostic factors in a model for survival from glioblastoma multiforme: an observational study of a cohort of consecutive non-selected patients from a single institution. BMC Cancer 13, 402 (2013). 
46. Gorlia T, Stupp R, Brandes AA et al. New prognostic factors and calculators for outcome prediction in patients with recurrent glioblastoma: a pooled analysis of EORTC Brain Tumour Group Phase I and II clinical trials. Eur. J. Cancer 48(8), 1176-1184 (2012).

47. Chinot OL, Wick W, Mason W et al. Bevacizumab plus radiotherapy-temozolomide for newly diagnosed glioblastoma. N. Engl. J .Med. 370(8), 709-722 (2014).

48. Recht L, Mechtler LL, Wong ET, O'Connor PC, Rodda BE. Steroid-sparing effect of corticorelin acetate in peritumoral cerebral edema is associated with improvement in steroid-induced myopathy. J. Clin. Oncol. 31(9), 1182-1187 (2013).

49. Arvold ND, Armstrong TS, Warren KE et al. Corticosteroid use endpoints in neuro-oncology: Response Assessment in Neuro-Oncology working group. Neuro Oncol. 20(7), 897-906 (2018).

50. Albert NL, Weller M, Suchorska B et al. Response Assessment in Neuro-Oncology working group and European Association for Neuro-Oncology recommendations for the clinical use of PET imaging in gliomas. Neuro Oncol. 18(9), 1199-1208 (2016)

51. Sharma M, Juthani RG, Vogelbaum MA. Updated response assessment criteria for high-grade glioma: beyond the MacDonald criteria. Chin. Clin. Oncol. 6(4), 37 (2017).

52. Galldiks N, Albert NL, Sommerauer M et al. PET imaging in patients with meningioma-report of the RANO/PET group. Neuro Oncol. 19(12), 1576-1587 (2017).

53. D’Arco F, Culleton S, De Cocker LJL, Mankad K, Davila J, Tamrazi B. Current concepts in radiologic assessment of pediatric brain tumors during treatment, part 1. Pediatr Radiol.(2018). doi: 10.1007/s00247-018-4194-9[Epub aheadof print] Review. PubMed PMID: 29980859

-. Discusses the current challenges to radiographic assessment of disease and treatment response in pediatric brain tumors.

54. Warren KE, Poussaint TY, Vezina G et al. Challenges with defining response to antitumor agents in pediatric neuro-oncology: a report from the Response Assessment in Pediatric Neuro-Oncology (RAPNO) working group. Pediatr. Blood Cancer 60(9), 1397-1401 (2013).

55. Warren KE, Vezina G, Poussaint TY et al. Response assessment in medulloblastoma and leptomeningeal seeding tumors: recommendations from the Response Assessment in Pediatric Neuro-Oncology committee. Neuro Oncol. 20(1), 13-23 (2018).

-• Provides an excellent summary of initial recommendations for assessing disease and treatment response in medulloblastoma and similar tumors. 
